\title{
Perforin: an important player in immune response
}

\author{
IWONA OSINSSKA ${ }^{1,2}$, KATARZYNA POPKO ${ }^{2}$, URSZULA DEMKOW ${ }^{2}$
}

${ }^{1} \mathrm{PhD}$ Study, Department of Pathology, Medical University of Warsaw, Poland

${ }^{2}$ Department of Laboratory Diagnostics and Clinical Immunology of Developmental Age, Medical University of Warsaw, Poland

\begin{abstract}
Perforin is a glycoprotein responsible for pore formation in cell membranes of target cells. Perforin is able to polymerize and form a channel in target cell membrane. Many research groups focus on the role of perforin in various diseases, immune response to bacterial and viral infections, immune surveillance and immunopathology. In addition, perforin is involved in the pathogenesis of autoimmune diseases and allogeneic transplant rejection. Natural killer (NK) cells and CD8-positive T-cells are the main source of perforin. However, CD4-positive T-cells are also able to express a low amount of perforin, when classic cytotoxicity is ineffective or disturbed.

Polymerized perforin molecules form channels enabling free, non-selective, passive transport of ions, water, small-molecule substances and enzymes. In consequence, the channels disrupt protective barrier of cell membrane and destroy integrity of the target cell. This review will focus on mechanisms of action and structure of perforin. Also, in this review we discuss the problem of abnormal perforin production in diseases such as: hemophagocytic lymphohistiocytosis (HLH), leukemias and lymphomas, infectious diseases and autoimmune diseases. Better understanding of the role of these molecules in health and disease will open a new field of research with possible therapeutic implications.
\end{abstract}

Key words: granzyme, cytolytic granules, HLH, type 1 diabetes, autoimmune diseases, SLE, Hashimoto's disease.

(Centr Eur J Immunol 2014; 39 (1): 109-115)

\section{Introduction}

Perforin is a glycoprotein responsible for pore formation in cell membranes of target cells. The note about the structure and function of human perforin was published in 1988 [1]. Its structure and mechanism of action was once again described in detail in a paper published in 2010 by researchers from the Monash University [2]. Such articles as these complemented the work of a Nobel prize winner - Jules Bordet, who 110 years ago published the first hypothesis on pore formation in target cells as a part of immune response.

Natural killer (NK) cells and CD8-positive T-cells are the main source of perforin [3]. However, CD4-positive T-cells are also able to express a low amount of perforin, when classic cytotoxicity is ineffective or disturbed [4].

Many research groups focus on the role of perforin in various diseases. Much attention is paid to their role in immune response to bacterial and viral infections, immune surveillance and immunopathology [5]. In addition, perforin is involved in the pathogenesis of autoimmune diseases and allogeneic transplant rejection [6].

\section{Structure of perforin}

Perforin is a $60-70-\mathrm{kDa}$ glycoprotein $[1,7]$. The National Protein Database indicates it has 555 amino acids, which should give it about $70 \mathrm{kDa}$. Single perforin molecule consists of four domains, including two $(N$-terminal and $C$-terminal) typical for perforin and related to its biological functions. The other two domains, which are located in the center of the molecule, are $20 \%$-homologous to analogous domains in complement molecules (C6, C7, C8 and C9). One of the homologous domains contains a sequence allowing formation of two $\beta$-sheets and one $\alpha$-helix structures. It is a hydrophobic domain able to incorporate into the lipid membrane of the target cell. In addition, a perforin molecule contains a cysteine-rich domain homologous to low density lipoprotein (LDL) receptor type $\mathrm{B}$ and epithelial growth factor (EGF) precursor. N-terminal domain of perforin contains $\mathrm{Ca}^{2+}$ ions binding site that is related to its biological function $[2,8]$.

The pores formed by perforin in the target cell are 5-20 $\mathrm{nm}$ in diameter [9]. A 14-nm channel is formed by approximately 20 perforin molecules; however, it was shown that

Correspondence: Iwona Osińska, MSc, Department of Laboratory Diagnostics and Clinical Immunology of Developmental Age, Medical University of Warsaw, Marszałkowska 24, 00-576 Warsaw, Poland, e-mail: osinskaiwi@gmail.com 


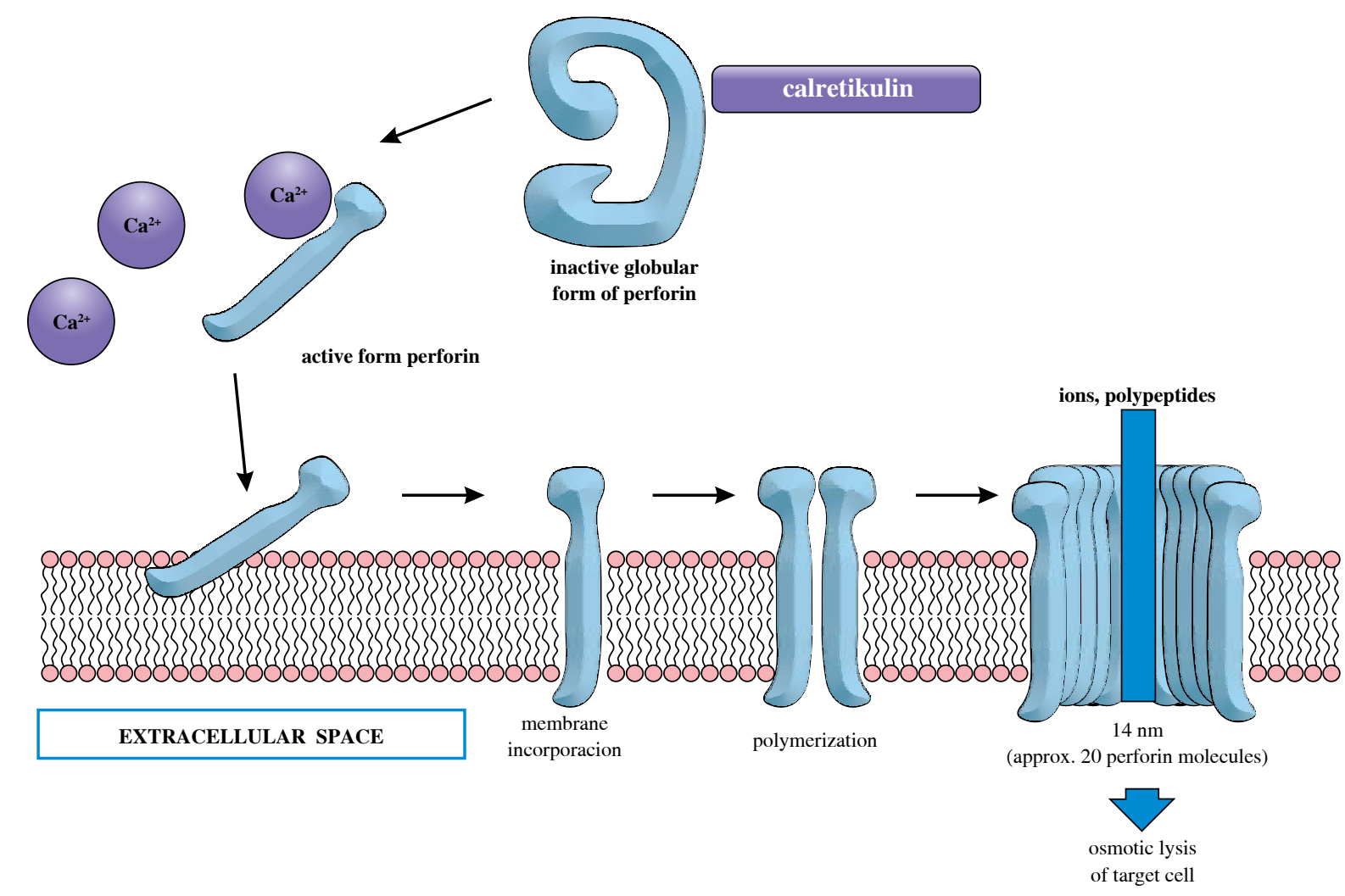

Fig. 1. Formation of channel in a target cell membrane (according to $[8,9]$ - modified)

even 3-4 perforin molecules are able to form an efficient pore (Fig. 1) [8]. Polymerized perforin molecules form cylindrical, hydrophobic channels enabling free, non-selective, passive transport of ions, water, small-molecule substances and enzymes. In consequence, the channels disrupt protective barrier of cell membrane and destroy integrity of the target cell [9].

\section{Perforin - mechanism of action}

Perforin is able to polymerize and form a channel in the target cell membrane. Perforin polymerization and pore formation require $\mathrm{Ca}^{2+}$ ions [7]. They are responsible for transformation of the inactive, globular form to active perforin able to incorporate into cell membrane (Fig. 1). Both incorporation and polymerization processes depend on various factors including temperature, phosphatidylserine content, platelet-activating factor (PAF) as well as activation of membrane receptors $[2,8]$.

Calreticulin is an important enzyme of cytolytic granules that serves as a chaperone protein for perforin protecting it from activation and degradation by keeping it in the glomerular form. It binds $\mathrm{Ca}^{2+}$ ions and inhibits spontaneous perforin polymerization inside the granules (Fig. 1) [10].

Perforin binds target cells through membrane phospholipids. Phosphatidylcholine is able to bind $\mathrm{Ca}^{2+}$ ions and increases perforin affinity to target cell membrane [8]. Pores formed by perforin disrupt cell membrane and allow free influx and efflux of ions and polypeptides. As a result, mineral homeostasis is disturbed and tonic shock develops. Indirectly, it induces activation of pro-apoptotic pathways and DNA degradation leading to cell death $[2,8]$.

Calcium ions might be also responsible for inhibition of perforin polymerization and are able to block transmembrane channels. Such phenomenon is observed in low $\mathrm{pH}$ and increased concentration of calcium ions. Other factors inhibiting perforin functions include the activity of protein $\mathrm{S}$. Protein $\mathrm{S}$ is a glycoprotein inhibiting lytic activity of complement components (membrane attacking complex). Due to the structural homology between perforin and C9 complement component, protein $\mathrm{S}$ is able to inhibit perforin functions. Protein $\mathrm{S}$ competitively binds to the perforin binding site on the target cell and therefore inhibits pore formation [11, 12].

\section{Natural killer and CD8 cells as a source of perforin}

Perforin plays an important role in cytotoxic activity of NK and cytotoxic CD8 T cells [3]. Both NK and CD8 cells use the same mechanisms to destroy target cells; however, activation pathways differ. Natural killer cells, contrary to 


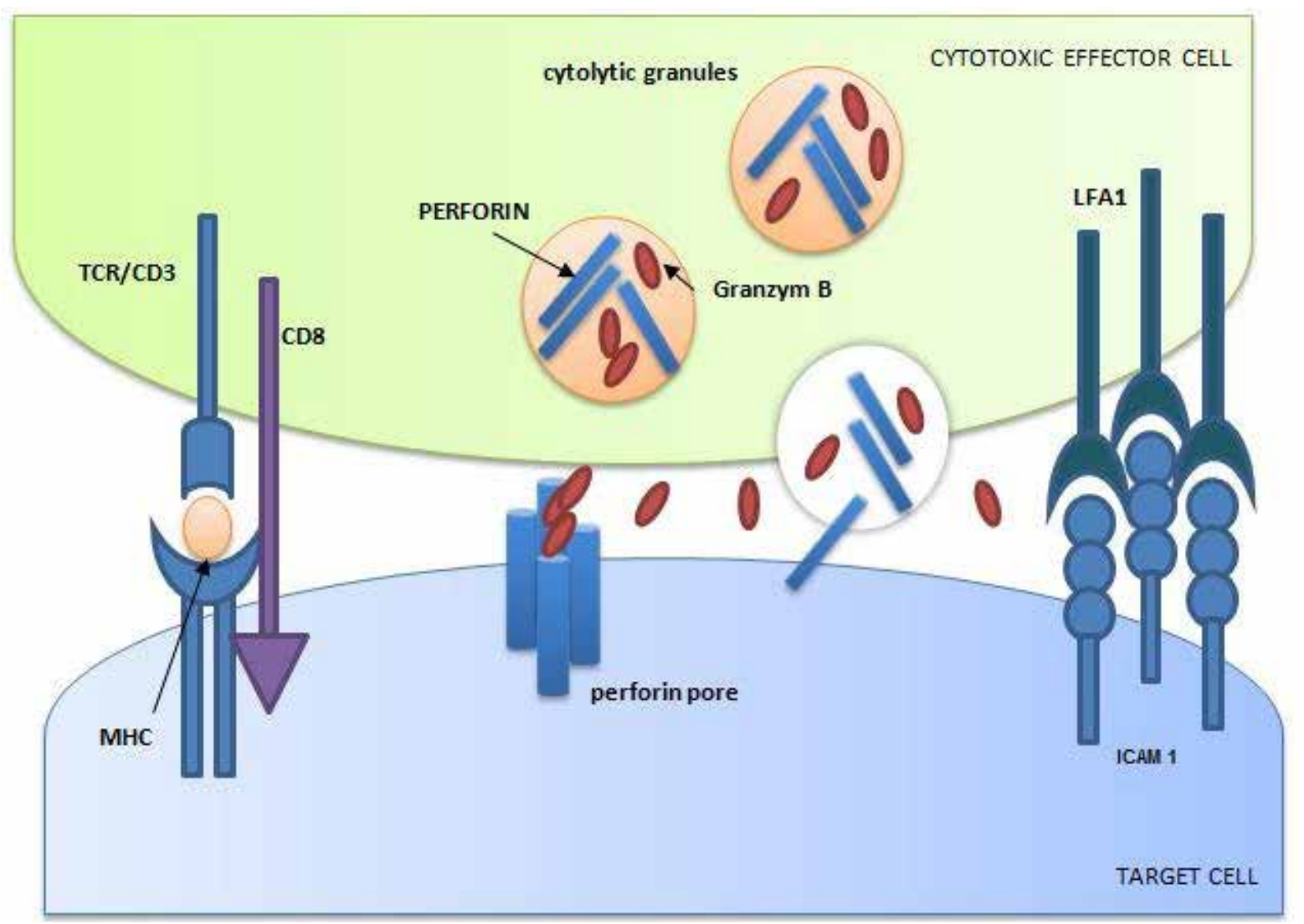

TCR - T-cell receptor, CD - cluster of differentiation, MHC-major histocompatibility complex, LFA1-lymphocyte function-associated antigen 1, ICAM 1 intercellular adhesion molecule 1

Fig. 2. Mechanism of action of cytotoxic effector cell (cytolytic granules) (according to [8, 13] - modified)

CD8 cells, exhibit spontaneous cytotoxicity towards target cells, which is dependent on perforin and granzyme B release [2]. The first step of the cytotoxic process is target cell recognition that can be specific (in the case of T CD8+ cells) and unspecific (in the case of NK cells). Unlike T cells, NK cells do not recognize MHC-bound antigens. The next step is formation of lytic synapse between the effector and target cell. The contact between these cells leads to the changes in the effector cell ultrastructure. Cell organelles (such as microtubules, Golgi apparatus and cytolytic granules) are translocated towards the synapse. The released granules contain perforin and granzymes B that are involved in cytotoxic reaction in target cells (Fig. 2) [8, 13].

Natural killer and cytotoxic T cells are resistant to the activity of other cytotoxic NK cells. Inhibition of pore formation in these cells is most probably related to reduced binding of perforin to the surface of their cell membrane $[14,15]$.

The best sensitive method of intracellular perforin detection is flow cytometry. Using the surface markers that identify specific cell populations, flow cytometry makes it possible to indentify expression of perforin on such cells at the same time. Other methods of detection of human perforin are ELISA, ELISpot and immunohistochemistry [16].

\section{Abnormal production or abnormal activity of perforin}

An important role of perforin in the immune system function was confirmed unequivocally in a mice model. Perforin knock-out mice had an abnormal function of the immune system or developed autoimmune diseases and lymphomas [8].

Perforin released from cytolytic granule binds to target cell membrane and form a pore leading to activation of cell cytotoxicity. Abnormal binding of perforin to the surface of the target cell is responsible for resistance to cytotoxic activity of effector cells (NK and CD8). Target cells show various escape mechanisms protecting against perforin activity. Cytotoxic effect can be also completely inhibited by anti-perforin antibodies [17].

The following diseases are characterized by the defect in production or activity of perforin: hemophagocytic lym- 
phohistiocytosis (HLH), leukemias and lymphomas, infectious diseases, autoimmune diseases.

\section{Hemophagocytic lymphohistiocytosis}

Hemophagocytic lymphohistiocytosis (HLH) is a disorder characterized by severe abnormalities related to uncontrollable hyperactivation of the immune system. There are two major types of HLH: primary, recessive autosomal disorder (familial hemophagocytic lymphohistiocytosis - FLH) and secondary HLH observed in the course of various diseases, e.g. viral infection, au- toimmune diseases, cancer or after chemotherapy. In physiological conditions, Th1-mediated immune response leads to activation of macrophages and simultaneous activation of cytotoxic T lymphocytes and NK cells that are responsible for silencing the excessive immune response (Fig. 3A). The immune response in the course of HLH is not inhibited, which results in the release of excessive amounts of pro-inflammatory cytokines [tumor necrosis factor (TNF), interleukina (IL)-1, IL-6, interferon $\gamma($ IFN- $\gamma$ )]. In consequence, potentially fatal metabolic abnormalities, organ failure and cytopenia

A immune reaction silencing

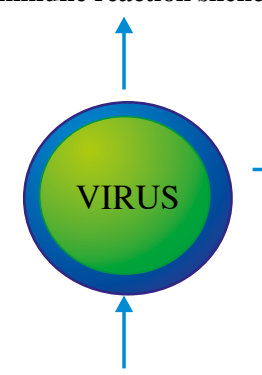

elimination

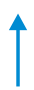

activation of cytotoxic T lymphocytes and NK cells

\section{PHYSIOLOGICAL CONDITIONS}

B

HEMOPHAGOCYTIC LYMPHOHISTIOCYTOSIS

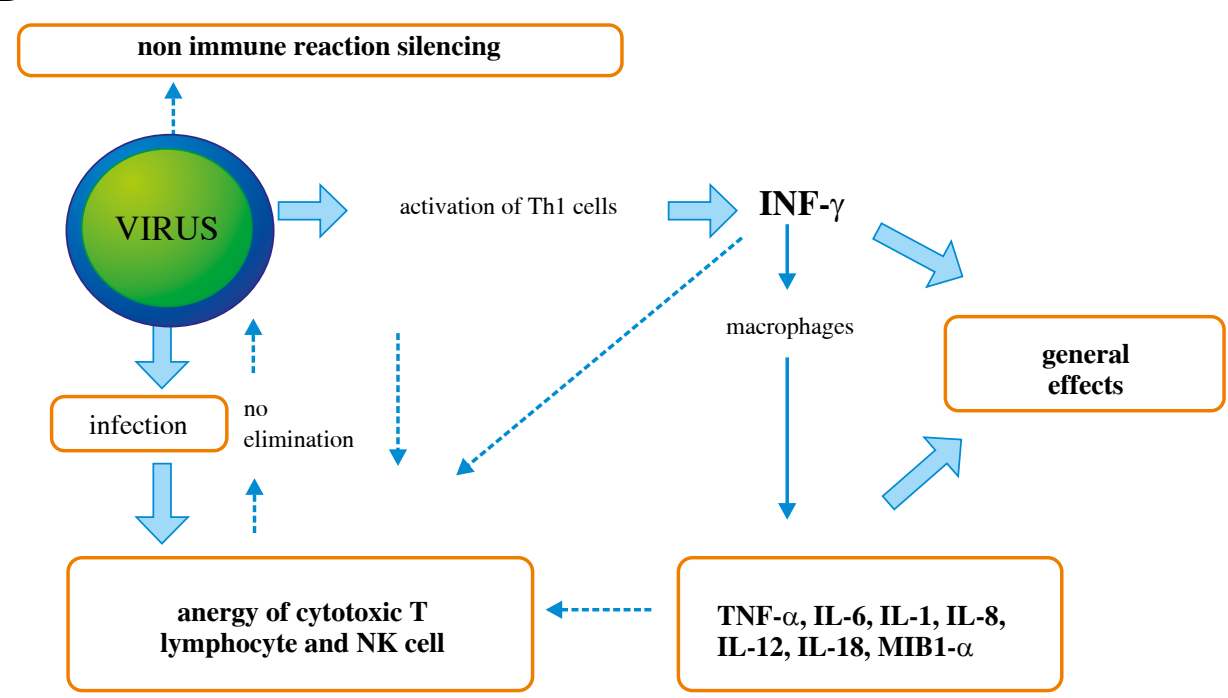

TNF-tumor necrosis factor, MIB1- $\alpha$ - mindbomb homolog 1 (gene), INF- $\gamma$-interferon $\gamma, N K$ - natural killer, HLH-hemophagocytic lymphohistiocytosis

Fig. 3. Immune response (according to [18] - modified): A) Normal immune response to viral infection, B) Pathologic immune response in HLH 
are observed (Fig. 3B) [18]. A typical clinical manifestation of HLH involves fever, hepatomegaly, splenomegaly and pancytopenia. Familial HLH usually manifests during the first months of life [3, 19].

Familial HLH might be caused by characteristic perforin gene mutations $[19,20]$. It proves that genetic defects leading to perforin deficiency may be a significant cause of HLH development. Because of that, the expression of perforin in CD16+/CD56+ NK cells was included in the diagnostic panel of HLH.

\section{Leukemias and lymphomas}

Perforin mutation leading to a complete deficiency of the protein is the cause of HLH. However, a partial deficiency in perforin production might be the cause of increased susceptibility to hematological malignancies (leukemias and lymphomas). A detailed analysis of patients with PRF1 mutations, with delayed symptoms typical of HLH, showed that half of the studied patients developed primary hematological malignancy [3].

Cytometric analyses revealed that abnormal binding of perforin to the target cell (neoplastic cell) is a major mechanism of resistance to perforin [17]. Mutation in both alleles of the perforin gene might be a cause of uncontrolled lymphoproliferation [21]. The studies performed in a model of perforin-deficient mice showed that these animals are significantly more susceptible to neoplastic diseases than those with normal perforin content. It confirms a significant role of perforin-dependent cytotoxic mechanisms in anti-tumor response [22, 23]. The role of perforin and granzymes B in inhibition of tumor growth and disease progression suggests that this mechanism may become a therapeutical target in the future [24].

\section{Infectious diseases}

Anti-viral activity of NK cells and cytotoxic T lymphocytes is based on the recognition and lysis of the target cell. It was shown that perforin-dependent cytotoxicity is an important element of response to acute infection of noncytopathic viruses, such as Lymphocytic Choriomeningitis Virus (LCMV). In the case of perforin deficiency, high titers of virus activate CTLs that are unable to eliminate the infection. In addition, constant production of proinflammatory cytokines is observed. This phenomenon was not observed for cytopathic viruses, such as Vaccinia virus (VACV) or Vesicular stomatitis virus (VSV). Infection caused by these viruses is controlled with other cytotoxic mechanisms [5]. The only exception is the response to Ebola virus infection, that is significantly dependent on perforin [25].

A key role of perforin was observed in Epstein-Barr virus (EBV) infections. In the acute and chronic course of mononucleosis, some patients exerted a decreased expression of perforin caused by mutation in both alleles of the perforin gene. The genetic defect may also lead to abnormalities in perforin structure and inhibition of perforin activation disabling the molecule from binding to target cell membrane [21].

Intracellular bacterial infections, including Listeria monocytogenes and Mycobacterium tuberculosis, are controlled by several effector mechanisms. Therefore, infection can be still controlled even in the case of perforin deficiency. However, in such cases the response is delayed, which proves an important role of perforin in response to primary infection with intracellular bacteria [5].

\section{Autoimmune diseases}

The role of perforin is demonstrated also in the pathogenesis of autoimmune diseases. The expression of perforin was studied in a group of children with Hashimoto's disease and in a group of healthy children by the flow cytometry method (our unpublished observations). The lower expression of perforin in CD8+ and NK populations was found in children with Hashimoto's disease in comparison to the control population ( $p=0.01$ and $p=0.004$, respectively). There was also a significant correlation between perforin expression in CD8+ lymphocytes and in NK cells in the study population. Our results confirm the role of perforin in the pathogenesis of autoimmune Hashimoto's thyroiditis. However, a limited number of patients involved in the study requires further research. Partial compliance with the our research was shown by $\mathrm{Wu}$ and colleagues [26]. They focused on the role of perforin in autoimmune thyroid diseases and studied the expression of perforin in lymphocytes infiltrating the thyroid gland in patients with Graves' disease and Hashimoto's disease patients and at the same time analysed the phenotype of cells containing perforin. In this study, the highest count of infiltrating $\mathrm{T}$ cells, which showed only a small part of activation properties was found in the thyroid. The predominant population was CD8 subpopulation with the expression of perforin, while the CD4 cells with expression of perforin were found only in patients with autoimmune Hashimoto's thyroiditis.

Another autoimmune disease with a likely role of perforin is systemic lupus erythematosus (SLE). Park and co-workers showed that in SLE, similarly to patients with Hashimoto's disease, the cytotoxicity is reduced, reported to affect the secretion of perforin and granzymes [27]. However, researchers have shown that in SLE, there is a decrease in the number of NK cells, which are responsible for the cytotoxicity. In contrast to our observations, where there was no decrease in the number of NK cells, authors did not find any difference in the number of CD8 and CD4 cells. Thus, it can be hypothesized that the malfunction or reduced expression of perforin in these cells is responsible for cytotoxicity disorders, not the decrease in the number of cells.

However, the reduced expression of perforin in CD8 and NK cells is not confirmed in other diseases of auto- 
A

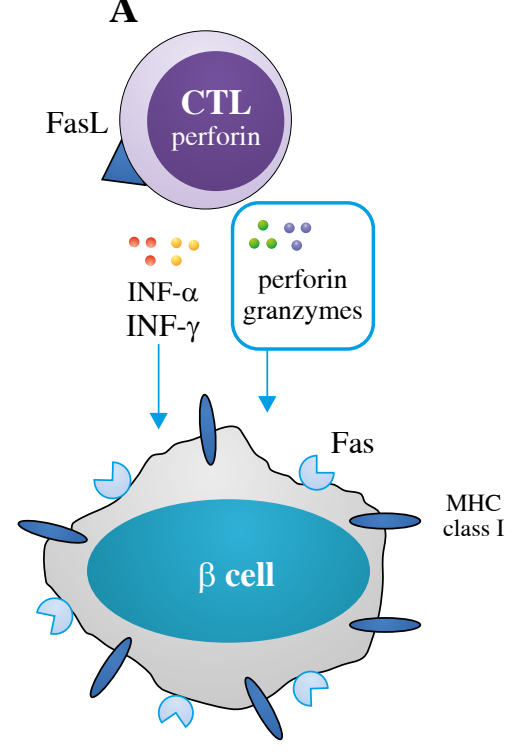

Perforin - dependent pathway
B

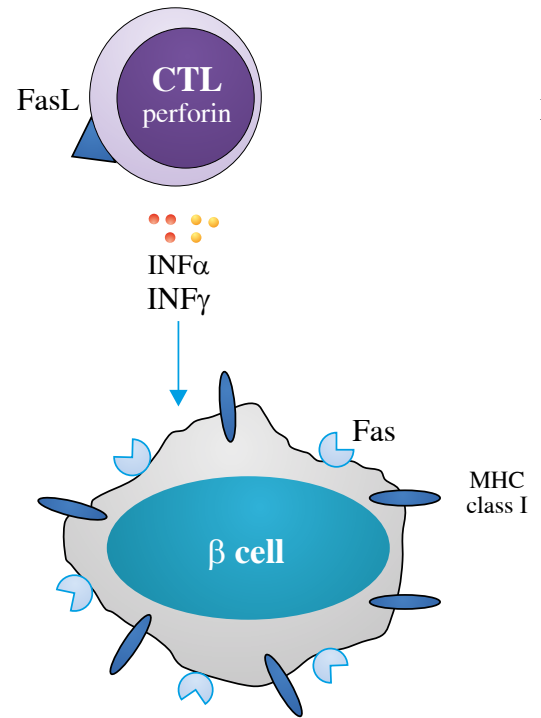

FasL - dependent pathway (no peforin)

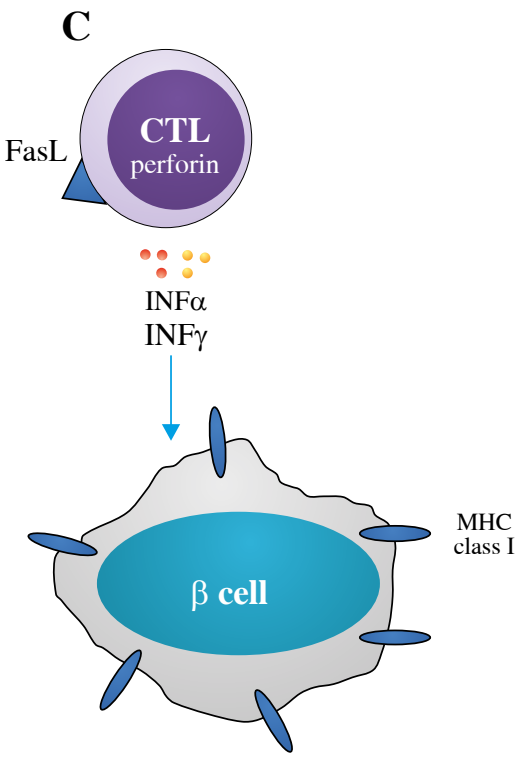

No killing (no perforin, no Fas)

Fas - Fas receptor, FasL - Fas ligand, CTLs - cytotoxic lymphocytes, INF- $\gamma$ - interferon $\gamma$, MHC-major histocompatibility complex, TNF - tumor necrosis factor

Fig. 4. The mechanisms of pancreatic $\beta$-cell destruction (according to [28] - modified): A) perforin and granzyme-dependent pathway; B) Fas/FasL interaction-dependent pathway (in case of perforin deficiency, CTLs use this method of apoptosis induction); C) cell survival (no perforin or Fas deficiency on $\beta$ cells) - in vitro conditions

immune origin. In type 1 diabetes, the perforin expression is excessive in contrast to patients with Hashimoto's disease or SLE. Studies performed using a mice model of the disease confirmed the role of cytotoxic $\mathrm{T}$ cells in the destruction of pancreatic $\beta$ cells [28]. Pancreatic $\beta$ cells may be destroyed in perforin/granzyme B-dependent pathway, Fas/FasL-dependent pathway as well as in a consequence of proinflammatory cytokines release (interferon, interleukins). It was shown that more than one cytotoxic mechanism is involved in $\beta$ cells destruction. If one of the mechanisms is blocked, the other replaces it. In addition, the main pathway responsible for $\beta$ cells destruction in the course of type 1 diabetes is related to perforin release by CD8 cells. In the case of perforin deficiency, this pathway is replaced by Fas/FasL pathway activation (Fig. 4).

\section{Conclusions}

The results of many studies confirm the important role of perforin in the pathogenesis of different diseases such as HLH, leukemias and lymphomas, infectious as well as autoimmune diseases. The structure of perforin and its function were widely evaluated. The methods of identification of such molecule are available. Recently the practical use of the results of these studies with possible treatment implication needs further studies.

Authors declare no conflict of interest.

\section{References}

1. Lichtenheld MG, Olsen KJ, Lu P, et al. (1988): Structure and function of human perforin. Nature 335: 448-451.

2. Law RH, Lukoyanova N, Voskoboinik I, et al. (2010): The structural basis for membrane binding and pore formation by lymphocyte perforin. Nature 468: 447-451.

3. Voskoboinik I, Dunstone MA, Baran K, et al. (2010): Perforin: structure, function, and role in human immunopathology. Immunol Rev 235: 35-54.

4. Williams NS, Engelhard VH (1997): Perforin-dependent cytotoxic activity and lymphokine secretion by CD4+ T cells are regulated by CD8+ T cells. J Immunol 159: 2091-2099.

5. van den Broek MF, Hengartner H (2000): The role of perforin in infections and tumour surveillance. Exp Physiol 85: 681-685.

6. Trapani JA, Smyth MJ (2002): Functional significance of the perforin/granzyme cell death pathway. Nat Rev Immunol 2: 735-747.

7. Young JD, Liu CC, Persechini PM (1988): Molecular mechanisms of lymphocyte-mediated killing. Braz J Med Biol Res 21: 1145-1153.

8. Lasek W, Malejczyk J (2007): Mechanizmy cytotoksyczności limfocytów. In: Immunologia. Gołąb J, Jakóbisiak M, Lasek W, Stokłosa T (eds.). PWN, Warszawa; 241-249.

9. Liu CC1, Young LH, Young JD (1996): Lymphocyte-mediated cytolysis and disease. N Engl J Med 335: 1651-1659.

10. Andrin C1, Pinkoski MJ, Burns K et al. (1998): Interaction between a $\mathrm{Ca}^{2+}$-binding protein calreticulin and perforin, a component of the cytotoxic T-cell granules. Biochemistry 37: 10386-10394.

11. Morgan BP, Harris CL (1999): Regulation in the terminal pathway. In: Complement regulatory proteins. Academic Press, Cambridge; 143. 
12. Tschopp J, Masson D, Schäfer S, et al. (1988): The heparin binding domain of S-protein/vitronectin binds to complement components $\mathrm{C} 7, \mathrm{C} 8$, and $\mathrm{C} 9$ and perforin from cytolytic T-cells and inhibits their lytic activities. Biochemistry 27: 4103-4109.

13. Voskoboinik I, Smyth MJ, Trapani JA (2006): Perforin-mediated target-cell death and immune homeostasis. Nat Rev Immunol 6: 940-952.

14. Jiang SB, Ojcius DM, Young JD (1990): Perforin binding to cells and lipid membranes determined by a simple competition assay. J Immunol Methods 126: 29-37.

15. Liu CC, Jiang S, Persechini PM, et al. (1989): Resistance of cytolytic lymphocytes to perforin-mediated killing. Induction of resistance correlates with increase in cytotoxicity. J Exp Med 169: 2211-2225.

16. Zuber B, Levitsky V, Jönsson G, et al. (2005): Detection of human perforin by ELISpot and ELISA: ex vivo identification of virus-specific cells. J Immunol Methods 302: 13-25.

17. Lehmann C, Zeis M, Schmitz N, Uharek L (2000): Impaired binding of perforin on the surface of tumor cells is a cause of target cell resistance against cytotoxic effector cells. Blood 96: 594-600.

18. Jędrzejczak WW (2008): Limfohistocytoza hemofagocytarna - rzadko rozpoznawany uleczalny stan bezpośredniego zagrożenia życia występujący również u dorosłych. Acta Heamato Pol 39: 515-526.

19. Kogawa K, Lee SM, Villanueva J, et al. (2002): Perforin expression in cytotoxic lymphocytes from patients with hemophagocytic lymphohistiocytosis and their family members. Blood 99: 61-66.

20. Stepp SE, Dufourcq-Lagelouse R, Le Deist F, et al. (1999): Perforin gene defects in familial hemophagocytic lymphohistiocytosis. Science 286: 1957-1959.

21. Katano H, Ali MA, Patera AC, et al. (2004): Chronic active Epstein-Barr virus infection associated with mutations in perforin that impair its maturation. Blood 103: 1244-1252.

22. Kägi D, Ledermann B, Bürki K, et al. (1994): Cytotoxicity mediated by $\mathrm{T}$ cells and natural killer cells is greatly impaired in perforin-deficient mice. Nature 369: 31-37.

23. van den Broek ME, Kägi D, Ossendorp F, et al. (1996): Decreased tumor surveillance in perforin-deficient mice. J Exp Med 184: 1781-1790.

24. Rousalova I, Krepela E (2010): Granzyme B-induced apoptosis in cancer cells and its regulation. Int J Oncol 37: 13611378.

25. Gupta M, Greer P, Mahanty S, et al. (2005): CD8-mediated protection against Ebola virus infection is perforin dependent. J Immunol 174: 4198-4202.

26. Wu Z, Podack ER, McKenzie JM, et al. (1994): Perforin expression by thyroid-infiltrating $T$ cells in autoimmune thyroid disease. Clin Exp Immunol 98: 470-477.

27. Park YW, Kee SJ, Cho YN, et al. (2009): Imparied differentation and cytotoxicity of natural killer cells in systemic lupus erythematosus. Arthritis Rheum 60: 1753-1763.

28. Thomas HE, Trapani JA, Kay TW (2010): The role of perforin and granzymes in diabetes. Cell Death Differ 17: 577-585. 